\title{
Interpretation of the infrared continuum in a solar white-light flare
}

\author{
M. D. Ding, Y. Liu, C.-T. Yeh, and J. P. Li \\ Department of Astronomy, Nanjing University, Nanjing 210093, PR China \\ Received 11 April 2002 / Accepted 17 March 2003

\begin{abstract}
We explain the origin of the infrared continuum in the solar flare of 10 March 2001 which shows a positive contrast in the maximum phase but possibly a negative contrast in the early phase. The general feature is consistent with a flare model heated by an electron beam. By making non-LTE model calculations, we find that in the early phase, when the electron then reduces the emergent intensity. With the flare development, the atmosphere is gradually heated. In particular, radiative backwarming plays a chief role in the heating of the temperature minimum region and upper photosphere. We estimate the temperature rise in these regions for a fully heated atmosphere in which energy balance is attained. In this case, the continuum emission rises above the quiescent value. Therefore, this corresponds to the flare maximum phase. We further find that the energy flux of the electron beam deduced from the hard X-ray emission is large enough to account for the continuum contrast.
\end{abstract} \\ beam bombards an unheated atmosphere, the non-thermal ionization by the beam results in an increased $\mathrm{H}^{-}$opacity which
}

Key words. Sun: atmosphere - Sun: flares - Sun: infrared

\section{Introduction}

In solar flare research, the heating of the lower atmosphere remains an interesting topic since it poses a constraint on the flare energetics and is related to the enhancement of line and continuum emission. In past decades, various energy transport mechanisms have been proposed to deposit energy and heat the layers in the temperature minimum region (TMR) and the upper photosphere. Those include heating by a non-thermal electron beam (e.g., Aboudarham \& Hénoux 1986) or a proton beam (Machado et al. 1978), soft X-ray irradiation (e.g., Hénoux \& Nakagawa 1977), EUV irradiation (e.g., Machado et al. 1978), dissipation of Alfvén waves (Emslie \& Sturrock 1982), and chromospheric radiative backwarming (Machado et al. 1989; Metcalf et al. 1990). However, most heating mechanisms, if working alone, are shown to be insufficient in producing the heating extent to explain observations (e.g., Machado et al. 1978; Emslie \& Machado 1979).

The problem become severer when interpreting the observations of some white-light flares (WLFs). A WLF is usually characterized by an enhanced emission at the Balmer or Paschen continuum. Recently, Liu et al. (2001) found that in a WLF of 10 March 2001, the near infrared (Brackett) continuum was increased relative to the quiescent level. This continuum is formed in the lower chromosphere or upper photosphere. The close relationship between the time history of $\mathrm{H} \alpha$ emission and that of hard X-ray emission implies that non-thermal electrons are the main heating agent in this flare. An electron beam

Send offprint requests to: M. D. Ding, e-mail: dmd@nju. edu.cn can induce an enhanced line emission of, for example, Balmer lines. As will be shown below, the role of an electron beam in the optical and near infrared continuum is less effective than in the lines. Therefore, it is clear that to fully account for the continuum emission in the flare of 10 March 2001, an appreciable temperature rise in the TMR and upper photosphere is required. Direct collisional heating by an electron beam is hard to produce this temperature rise since only $\gtrsim 200 \mathrm{keV}$ electrons can reach a column depth of $0.05 \mathrm{~g} \mathrm{~cm}^{-2}$ which corresponds to the TMR in the quiet-Sun atmosphere (Vernazza et al. 1981). Obviously, such electrons carry only a very small fraction of beam energy if assuming a low-energy cut-off of $20 \mathrm{keV}$. If not considering some in situ energy sources, the heating is most probably achieved through absorption of enhanced chromospheric radiation, i.e., radiative backwarming, as was previously proposed by Aboudarham \& Hénoux (1986).

On the other hand, observations indicated that flares on stars, for example, those on dMe stars, may in some cases be preceded by a continuum dip (e.g., Hawley et al. 1995). Such a dip is most plausibly explained by the absorption of photospheric radiation by an enhanced $\mathrm{H}^{-}$opacity (Grinin 1983), which could be related to the non-thermal ionization by an electron beam (Hénoux 1990; Ding \& Fang 2000). Hénoux (1990) further suggested that this phenomenon, called "blacklight flares" (BLFs), may also occur on the Sun and presented an observation of a solar flare which shows evidence of a negative contrast of up to $\sim 5 \%$ at $\lambda=5500 \AA$. Later on, van Driel-Gesztelyi et al. (1994) searched for BLFs in Yohkoh observed flares but found no unambiguous example. 


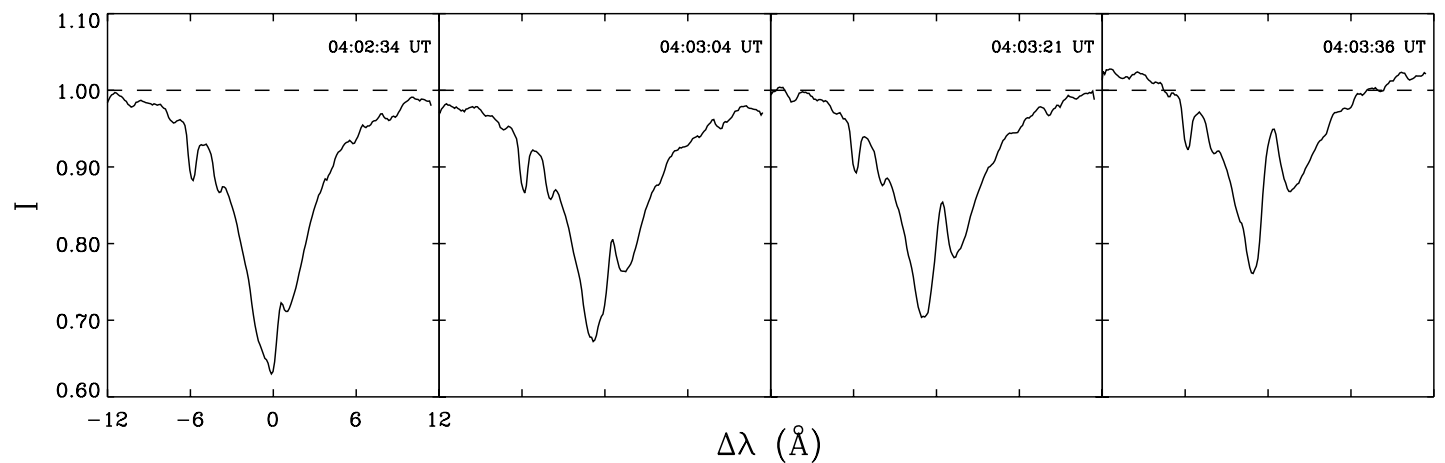

Fig. 1. Line profiles of Ca II $8542 \AA$ at four different times, showing the temporal variation of the line core emission and the nearby continuum. The profiles are normalized to the continuum intensity in the quiescent status. The four times are also shown as vertical bars in Fig. 6 for comparison.

The flare of 10 March 2001, however, can be regarded as another candidate of BLF since its light curve at the near infrared continuum shows a slight dip in the early phase of the flare (Liu et al. 2001). To show this clearly, we plot in Fig. 1 the line profiles of Ca II $8542 \AA$ at a specific point in the flaring region at four different times, the first one of which corresponds to the preflare phase, the second one to the early impulsive phase, while the later two to the impulsive phase. One notices that with the development of the flare, the line core shows a gradually increased net emission; the nearby continuum intensity (the intensity at far wings), however, is first reduced to some extent (04:03:04 UT), and then is enhanced (04:03:36 UT) relative to the quiescent value. The purpose of this paper is to provide a plausible explanation of the observed continuum in terms of the electron-heated flare model.

\section{Computational method and results}

\subsection{The effect of non-thermal excitation and ionization on the Brackett continuum}

An electron beam can cause non-thermal ionization and excitation of hydrogen atoms in the flaring atmosphere (Ricchiazzi \& Canfield 1983; Aboudarham \& Hénoux 1986, 1987; Fang et al. 1993; Kasparová \& Heinzel 2002). This effect redistributes the level populations with more atoms excited to higher levels or ionized. Accordingly, the hydrogen Balmer and Lyman lines are substantially enhanced (Fang et al. 1993; Hénoux et al. 1995); the Lyman continuum is enhanced which shows a lower color temperature relative to the thermal case (Ding \& Schleicher 1997); an appreciable enhancement can also appear in the Balmer continuum (e.g., Ding \& Fang 1996). All these lines and continua mentioned above are formed in higher layers where the beam electrons can penetrate. Continuum emission at the visible or the near infrared wavelengths is formed deeper (in the lower chromosphere and upper photosphere), so that the influence of the electron beam is less obvious. More importantly, in addition to the hydrogen recombination, the negative hydrogen ions $\left(\mathrm{H}^{-}\right)$play an important role in the emission in this spectral region.

We now evaluate quantitatively how an electron beam can alter the emission process at $\lambda=8500 \AA$. To this end, we select a model atmosphere similar to the F1 model of Machado et al. (1980) as representing the atmosphere prior to beam heating. We then solve the equations of radiative transfer and statistical equilibrium for this model atmosphere in two cases with and without including the non-thermal effects of an electron beam. In the former case, we need to include the non-thermal collisional rates in the statistical equilibrium equations. The method to calculate these rates is briefly described below.

Using the cold target approximation, the total rate of energy deposit of an electron beam at column depth $N$ is given by (Emslie 1978)

$H=K\left[\xi \Lambda+(1-\xi) \Lambda^{\prime}\right] n_{\mathrm{H}} \int_{E_{N}}^{\infty} \frac{F_{0}(E) \mathrm{d} E}{E\left(1-E_{N}^{2} / E^{2}\right)^{\frac{2+\beta}{4+\beta}}}$,

where $K=2 \pi e^{4}, F_{0}(E)$ is the initial flux spectrum of the electron beam, and

$E_{N}=\left[\left(2+\frac{\beta}{2}\right) \frac{\gamma K N}{\mu_{0}}\right]^{\frac{1}{2}}$

is the minimum energy of electrons that can penetrate to depth $N, \xi$ is the degree of ionization, and $\Lambda$ and $\Lambda^{\prime}$ are Coulomb logarithms for elastic and inelastic collisions, respectively. The cosine of the initial pitch angle, $\mu_{0}$, is taken to be unity here.

Therefore, the rate of energy deposit through inelastic collisions, which contributes to non-thermal excitation and ionization, is written as

$H^{\prime}=K(1-\xi) \Lambda^{\prime} n_{\mathrm{H}} \int_{E_{N}}^{\infty} \frac{F_{0}(E) \mathrm{d} E}{E\left(1-E_{N}^{2} / E^{2}\right)^{\frac{2+\beta}{4+\beta}}}$

The expressions for $\Lambda, \Lambda^{\prime}, \beta$, and $\gamma$ can be found in Emslie (1978). We assume a power law for the initial flux spectrum of the electron beam with a low-energy cut-off, which means

$F_{0}(E)= \begin{cases}A E^{-\delta}, & E>E_{1}, \\ 0, & E<E_{1},\end{cases}$

where $A=(\delta-2) \mathcal{F}_{1} E_{1}^{\delta-2} . \mathcal{F}_{1}$ is the energy flux of the electron beam.

We adopt an atomic model of four bound levels plus continuum for hydrogen. The non-thermal collisional excitation and 
ionization rates due to the bombarding electron beam are derived empirically using the collisional cross-sections for various transitions (Fang et al. 1993). The non-thermal rates are expressed as

$C_{i j}^{\mathrm{B}} \simeq a_{i j} H^{\prime} / n_{1}$,

where $n_{1}$ is the hydrogen ground level population. Only the transitions from the ground level are considered. In cgs units, we have the following coefficients: $a_{12}=2.94 \times 10^{10}, a_{13}=$ $5.35 \times 10^{9}, a_{14}=1.91 \times 10^{9}$, and $a_{1 c}=1.73 \times 10^{10}$.

The electron beam is assumed to have an energy flux $\mathcal{F}_{1}=$ $10^{11} \mathrm{ergs} \mathrm{cm}^{-2} \mathrm{~s}^{-1}$, a power index $\delta=4$, and a low-energy cutoff $E_{1}=20 \mathrm{keV}$. Figure 2 shows the column mass distribution of the source function and the total optical depth at $\lambda=8500 \AA$ in both cases with and without the electron beam bombardment. As usual, the source function is defined as $S_{v}=j_{v} / \chi_{v}$, where $j_{v}$ and $\chi_{v}$ are the emission and absorption coefficients, respectively; the optical depth is expressed as $\tau_{v}=\int_{0}^{z} \chi_{v} \mathrm{~d} z^{\prime}$, where $z$ is the geometrical depth measured from the top of the atmosphere. To check further how the continuum emission originates from the atmosphere, we also plot in Fig. 2 the contribution function to the intensity at $\lambda=8500 \AA$ from (1) the free-bound transition of $\mathrm{H}$ and (2) the free-bound and free-free transitions of $\mathrm{H}^{-}$. The contribution function (per unit geometrical depth) is calculated as

$C_{I, \mathrm{H}}=j_{v, \mathrm{H}} \exp \left(-\tau_{v} / \mu\right) / \mu$,

and

$C_{I, \mathrm{H}^{-}}=j_{v, \mathrm{H}^{-}} \exp \left(-\tau_{v} / \mu\right) / \mu$

where $j_{v, \mathrm{H}}$ and $j_{v, \mathrm{H}^{-}}$are the emission coefficients of $\mathrm{H}$ and $\mathrm{H}^{-}$, respectively. For the flare of 10 March 2001, $\mu=0.63$, which is adopted in the computations throughout the paper.

From the figure, we can judge the effect of the electron beam on the Brackett continuum. It can obviously increase the source function in the upper chromosphere, where the hydrogen photo-recombination rates are enhanced. However, the opacity of this region is too small to produce a significant amount of continuum emission. In the lower chromosphere and below, the source function remains almost unchanged. However, the optical depth is increased down to the upper photosphere. This increased opacity can enlarge the chromospheric contribution but simultaneously reduce the photospheric contribution to the continuum. The latter is predominant since the main contributor lies in the upper photosphere, as shown in Fig. 2 (the lower-right panel). This explains why the continuum emission at this wavelength decreases when non-thermal effects exist in an unheated atmosphere.

\subsection{The role of the backwarming effect}

As revealed above, the non-thermal effects of an electron beam cannot directly produce the observed continuum enhancement, which then requires a heating in the lower atmosphere. This heating can be achieved by radiative coupling. Non-thermal effects enhance the (Balmer and Paschen) continuum radiation
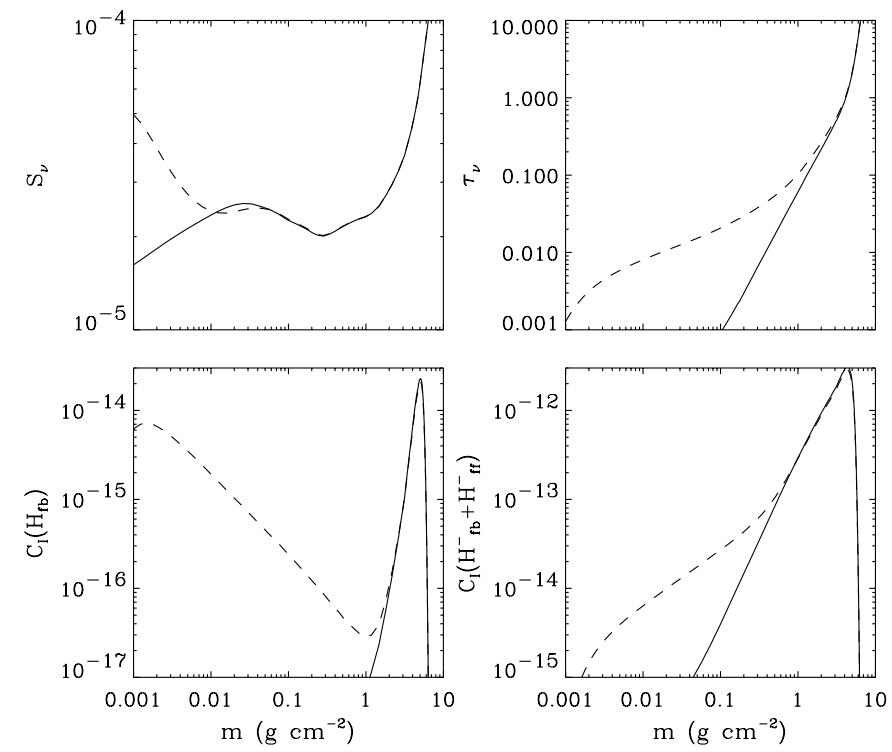

Fig. 2. Distributions of the source function, optical depth, and contribution function to the intensity at $\lambda=8500 \AA$ in the flare atmosphere. Quantities $S_{v}$ and $C_{I}$ are in cgs units. Solid lines refer to the case without electron beam bombardment, while dashed lines to the case with an electron beam $\left(\mathcal{F}_{1}=10^{11} \mathrm{ergs} \mathrm{cm}^{-2} \mathrm{~s}^{-1}, \delta=4\right.$, and $\left.E_{1}=20 \mathrm{keV}\right)$.

from the chromosphere, and meanwhile raise the $\mathrm{H}^{-}$population around the temperature minimum region. Therefore, this region can absorb more radiation energy from both the chromosphere and the photosphere; the temperature there must increase accordingly in order for the atmosphere to reach a new energy balance.

To evaluate quantitatively how the lower atmosphere can be radiatively heated in the presence of an electron beam, we adopt a similar method to that proposed by Aboudarham \& Hénoux (1987). We first calculate the radiative cooling rates in the background atmosphere,

$\Phi=4 \pi \int \chi_{v}\left(S_{v}-J_{v}\right) \mathrm{d} v$

where $J_{v}$ is the mean radiation intensity. The integration extends over all radiative transitions for the hydrogen atomic model adopted here. Cooling by the negative hydrogen ions is also included, which is important in the energy balance in the lower atmosphere. As above, we consider the two cases with and without a non-thermal electron beam. The results are plotted in Fig. 3. It is clear that in the non-thermal case, the cooling rate in the chromosphere becomes larger, the contribution of which is mainly from hydrogen bound-bound and free-bound transitions. The cooling rate in the photosphere, originally negative, now becomes more negative, which means an enhanced absorption of radiation by $\mathrm{H}^{-}$in these layers. However, what is important is the difference of the cooling rates in these two cases. If we think that in the thermal case, the background atmosphere is in energy balance, i.e., the non-zero cooling rates can be compensated for by some non-radiative heating/cooling processes, then in the non-thermal case, the reduced cooling rates in the lower atmosphere do imply a heating effect there, as expected. 


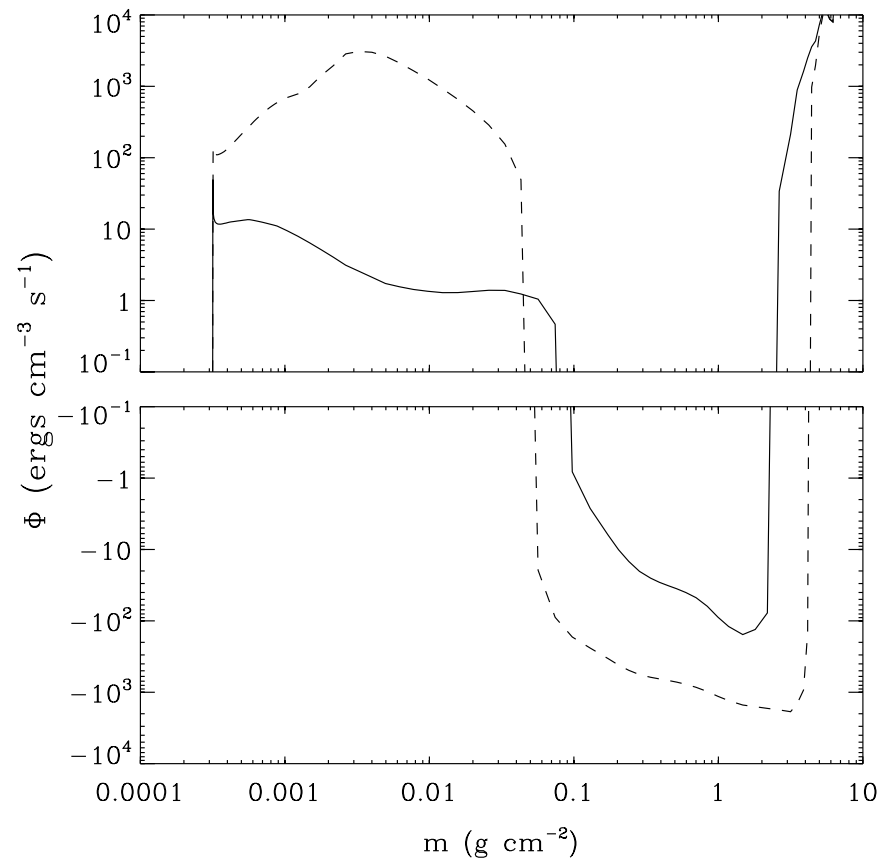

Fig. 3. Distribution of the total radiative cooling rate in the atmosphere. The solid line refers to the case without electron beam bombardment, while the dashed line to the case with an electron beam $\left(\mathcal{F}_{1}=10^{11} \mathrm{ergs} \mathrm{cm}^{-2} \mathrm{~s}^{-1}, \delta=4\right.$, and $\left.E_{1}=20 \mathrm{keV}\right)$, in which a marked difference is seen.

Therefore, we reconstruct the temperature distribution of the heated atmosphere under the constraint of energy balance, which means

$H=\Phi_{\mathrm{NT}}(T+\Delta T)-\Phi_{\mathrm{T}}(T)$,

where $H$ refers to the total heating rate by the electron beam, and $\Phi_{\mathrm{NT}}$ and $\Phi_{\mathrm{T}}$ are radiative cooling rates in the non-thermal and thermal cases, respectively. $\Delta T$ represents the modification of the temperature structure that is required for Eq. (9) to hold. In fact, in the lower atmosphere, $H$ is relatively small; $\Delta T$ is chosen such that $\Phi_{\mathrm{NT}}(T+\Delta T)$ approaches $\Phi_{\mathrm{T}}(T)$. Because of the radiative coupling between different layers, it is not a trivial thing to solve for $\Delta T$. Here, we do it semi-empirically. Note that in the computations of $\Phi$, we have not included other cooling sources than $\mathrm{H}$ and $\mathrm{H}^{-}$. This is fairly justified if we assume that the contribution of other elements changes much less significantly than that of $\mathrm{H}$ and $\mathrm{H}^{-}$from the thermal to non-thermal cases. Using the above method, we have obtained a series of modified model atmospheres corresponding to various electron beams, which are plotted in Fig. 4. We vary the energy flux of the beam but use a fixed low-energy cut-off, $E_{1}=20 \mathrm{keV}$.

From Fig. 4, one finds a significant temperature rise in the lower atmosphere in response to the backwarming effect. Note that such a case refers to the fully heated atmosphere which can only be reached if the heating persists for an enough long time, say, 15-25 s (Hénoux et al. 1990). There is also a temperature rise in the chromosphere, which is due to non-thermal collisional heating (elastic collisions) and also to radiative coupling between the photosphere and the chromosphere.

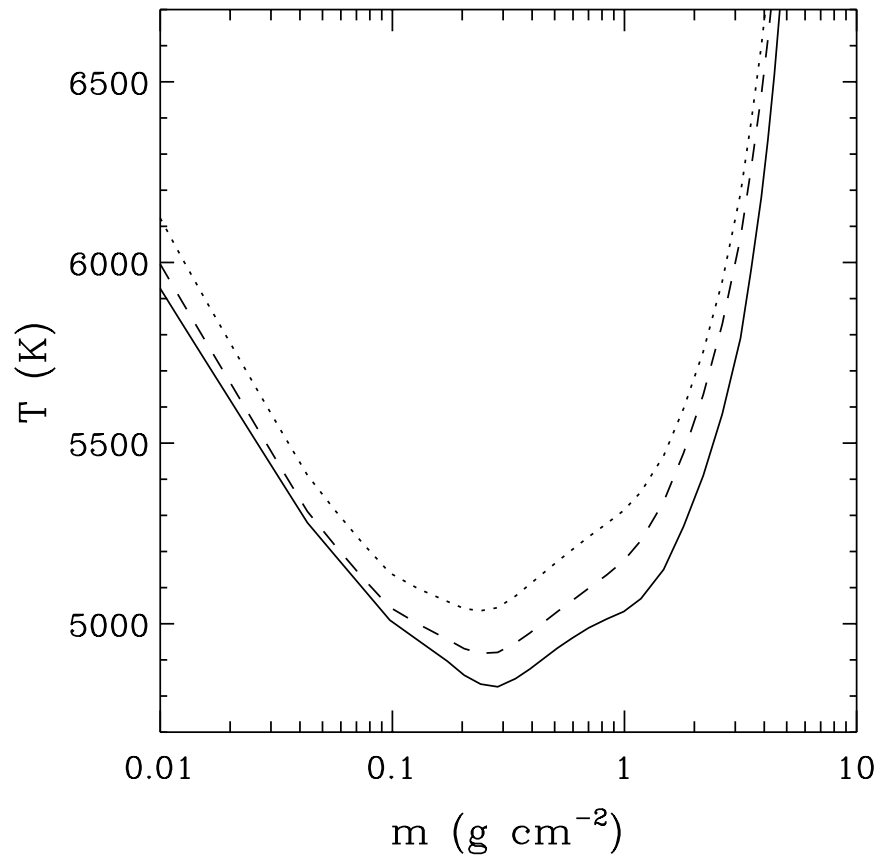

Fig. 4. Distribution of temperature for the atmosphere heated by electron beams of $\mathcal{F}_{1}=10^{10} \mathrm{ergs} \mathrm{cm}^{-2} \mathrm{~s}^{-1}$ (dashed line) and $10^{11} \mathrm{ergs} \mathrm{cm}^{-2} \mathrm{~s}^{-1}$ (dotted line). In both cases, $\delta=4$ and $E_{1}=$ $20 \mathrm{keV}$. The solid curve refers to the unheated atmosphere.

\subsection{Origin of the continuum emission}

We then explore the origin of the continuum emission at $\lambda=$ $8500 \AA$. We consider three cases corresponding to different stages in the flare development: (1) a background atmosphere without any electron beam, (2) a background atmosphere with a precipitating electron beam, and (3) a heated atmosphere with an electron beam. Here, the heated atmosphere refers to the one with energy balance obtained as above. These three cases can be roughly regarded as the preflare (quiescent) status, the early impulsive phase (the onset of beam heating), and the flare maximum phase, respectively. Denote by $I_{\mathrm{q}}$ the continuum intensity calculated in case (1), and $I_{\mathrm{f}}$ the continuum intensity in cases (2) and (3), the continuum contrast is defined as

$R=\left(I_{\mathrm{f}}-I_{\mathrm{q}}\right) / I_{\mathrm{q}}$.

Figure 5 plots the continuum contrast as a function of the energy flux of the beam.

\subsubsection{Case I: Continuum dip in the early phase}

The continuum contrast at $\lambda=8500 \AA$ observed in the flare of 10 March 2001 (Liu et al. 2001) appears negative in a small area at an early time. The maximum amplitude of continuum dip measured in a single pixel is $\sim 1-2 \%$. This point is located near the edge of the flare kernel, where the line profiles of Ca II $8542 \AA$ are shown in Fig. 1. Although this event cannot fully be confirmed to be a BLF since the amplitude of dip is not large enough to exclude the possible effect of measurement error, it is nevertheless consistent with the picture that in the early phase, an electron beam bombards an unheated atmosphere and causes a continuum dimming. This dimming 


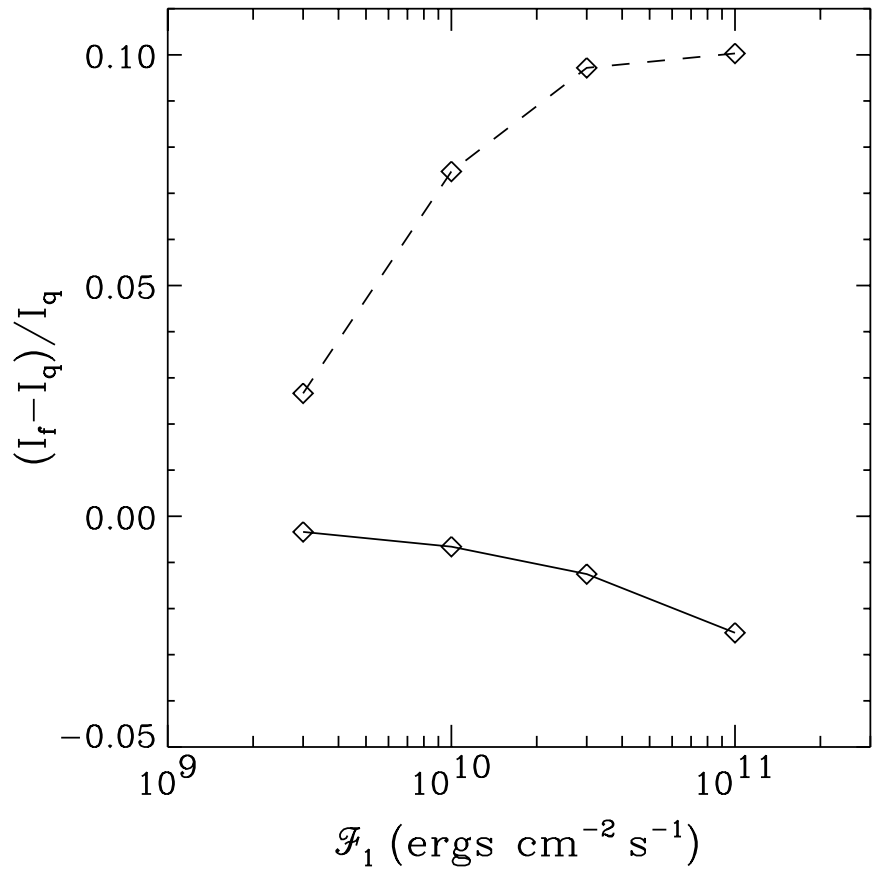

Fig. 5. Continuum contrast at $\lambda=8500 \AA$ varying with the energy flux of the electron beam ( $\delta=4$ and $\left.E_{1}=20 \mathrm{keV}\right)$. The curves show the continuum enhancement in cases of a background atmosphere with an electron beam (solid line) and a heated atmosphere with an electron beam (dashed line) relative to the quiescent status.

disappears soon after the atmosphere is heated both collisionally and radiatively.

\subsubsection{Case II: Continuum enhancement at the maximum time}

Observations also show that the continuum contrast increases to its maximum of $\sim 4 \%$ in $\sim 20 \mathrm{~s}$ from the onset of electron beam heating (judged from the time profile of hard X-ray emission, see Fig. 6). This time period is roughly enough for the lower atmosphere to get fully heated by the radiation from the chromosphere. From Fig. 5, we see that a beam with a flux of $\mathcal{F}_{1}=10^{10} \mathrm{ergs} \mathrm{cm}^{-2} \mathrm{~s}^{-1}$ can produce a continuum contrast of $\sim 7 \%$, already above the observed value. However, we note that the observed contrast represents a lower limit of the real contrast because of the limit of the spatial resolution.

\subsection{Observational constraints}

The hard X-ray telescope (HXT) of Yohkoh has well observed this flare. The hard X-ray flux in the first three energy channels $(14-23,23-33$, and $33-53 \mathrm{keV})$ is displayed in Fig. 6. We find that the 14-23 keV light curve has two main peaks, of which only the first peak has a corresponding peak in the two higher channels. It is interesting that the first peak is also roughly coincident with the peak of the infrared continuum emission and the $\mathrm{H} \alpha$ width (Liu et al. 2001), if taking into account the relatively low time resolution in the ground-based 2D spectral observations. We thus suggest that the first peak of hard X-ray emission is mainly of non-thermal origin (due to thick-target

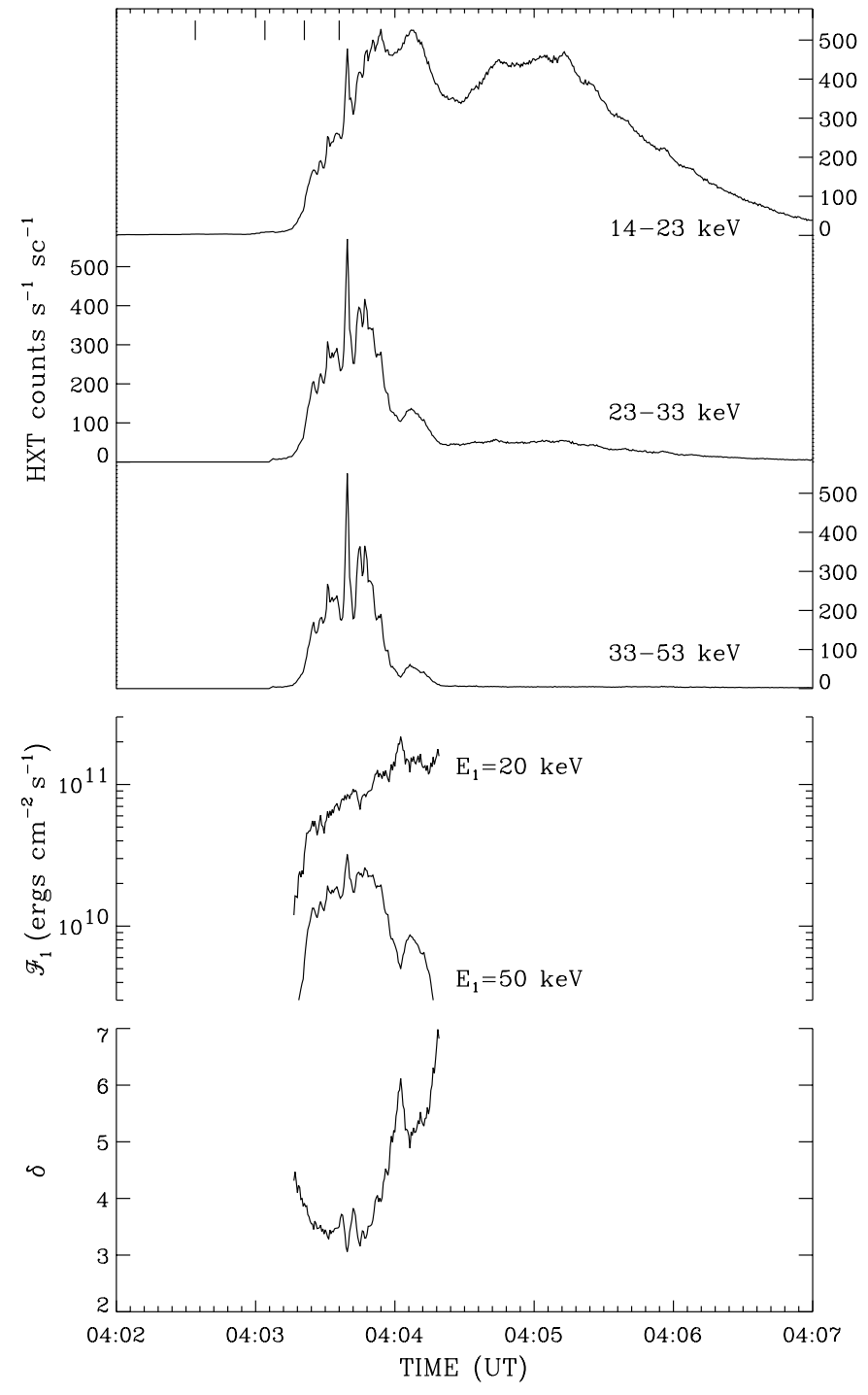

Fig. 6. Time profiles of HXR emission observed by Yohkoh and the deduced energy flux and power index of the non-thermal electron beam. The four vertical bars in the top panel correspond to the four times at which the line profiles of Ca II $8542 \AA$ are shown in Fig. 1 .

bremsstrahlung), which supports our assumption that energetic electrons are the main energy input to the flaring atmosphere during the impulsive phase.

Therefore, we use a similar method to that in Fang et al. (1998) in order to get the flux spectrum of the electron beam that produces the hard X-ray emission. To avoid the contamination of thermal bremsstrahlung, we use the 23-33 and 33$53 \mathrm{keV}$ flux curves to derive the power index and the energy flux of the beam. In this method, the key parameter is the total area with electron beam heating, which is difficult to determine. Since one of the most significant effects of a non-thermal electron beam is to broaden the $\mathrm{H} \alpha$ line (Fang et al. 1993), we arbitrarily attribute the profiles with a full width at half maximum exceeding $3 \AA$ as caused by non-thermal effects. The total area thus amounts to $1.13 \times 10^{18} \mathrm{~cm}^{2}$.

The deduced power index, $\delta$, and the energy flux of the beam, $\mathcal{F}_{1}$, are also shown in Fig. 6 . One finds that during the impulsive phase ( 04:03-04:04 UT), $\delta$ varies in the range 
of 3-6. In deriving the energy flux, we have adopted two values for the low-energy cut-off, $E_{1}=20 \mathrm{keV}$, as usually assumed, and $50 \mathrm{keV}$, as implied by a recent investigation by Gan et al. (2001) that the cut-off energy may be higher. In the first case, $\mathcal{F}_{1}$ reaches a maximum of $\gtrsim 10^{11} \mathrm{ergs} \mathrm{cm}^{-2} \mathrm{~s}^{-1}$ while it is about one order of magnitude lower in the second case. However, we note that in both cases, the energy flux is larger enough to explain the observed enhancement of continuum emission (see Fig. 5).

\section{Conclusions}

The flare of 10 March 2001 shows an enhanced emission at the Brackett continuum in the maximum phase and may be classified as a type I WLF (Liu et al. 2001). On the other hand, it also shows evidence of continuum dimming in an early phase, i.e., at the beginning of flare eruption. Therefore, this flare is among very few candidates of the BLFs that are supposed to exist on the Sun (Hénoux et al. 1990). We explain in this paper the origin of the continuum feature for this flare in terms of an electron-beam-heated flare model. The main points are as follows. In the early phase, an electron beam is accelerated to bombard an unheated atmosphere; the non-thermal ionization by the beam results in an increased $\mathrm{H}^{-}$opacity which then reduces the emergent intensity. With the flare development, the atmosphere is gradually heated. In this period, radiative backwarming is the chief heating agent in the TMR and upper photosphere. We further estimate the temperature rise in these regions for a fully heated atmosphere in which energy balance is attained. This case corresponds roughly to the maximum phase, in which the continuum emission rises naturally above the quiescent value, consistent with what is observed. Based on the hard X-ray emission observed by Yohkoh, we deduce the energy flux of the non-thermal electron beam in the impulsive phase, which is shown to be large enough to account for the continuum contrast.
Acknowledgements. We thank the referee for valuable comments. This work was supported by TRAPOYT, NKBRSF under grant G20000784, and National Natural Science Foundation of China (NSFC) under grant 10025315.

\section{References}

Aboudarham, J., \& Hénoux, J.-C. 1986, A\&A, 168, 301 Aboudarham, J., \& Hénoux, J.-C. 1987, A\&A, 174, 270

Ding, M. D., \& Fang, C. 1996, A\&A, 314, 643

Ding, M. D., \& Fang, C. 2000, MNRAS, 317, 867

Ding, M. D., \& Schleicher, H. 1997, A\&A, 322, 674

Emslie, A. G. 1978, ApJ, 224, 241

Emslie, A. G., \& Machado, M. E. 1979, Sol. Phys., 64, 129

Emslie, A. G., \& Sturrock, P. A. 1982, Sol. Phys., 80, 99

Fang, C., Hénoux, J.-C., \& Gan, W. Q. 1993, A\&A, 274, 917

Fang, C., Tang, Y. H., Hénoux, J.-C., et al. 1998, Sol. Phys., 182, 163

Gan, W. Q., Li, Y. P., \& Chang, J. 2001, ApJ, 552, 858

Grinin, V. P. 1983, in Activity in Red Dwarf Stars, ed. P. B. Byrne, \& M. Rodonò (Dordrecht: Reidel), 613

Hawley, S. L., Fisher, G. H., Simon, T., et al. 1995, ApJ, 453, 464

Hénoux, J.-C., Aboudarham, J., Brown, J. C., et al. 1990, A\&A, 233, 577

Hénoux, J.-C., Fang, C., \& Gan, W. Q. 1995, A\&A, 297, 574

Hénoux, J.-C., \& Nakagawa, Y. 1977, A\&A, 57, 105

Kasparová, J., \& Heinzel, P. 2002, A\&A, 382, 688

Liu, Y., Ding, M. D., \& Fang, C. 2001, ApJ, 563, L169

Machado, M. E., Emslie, A. G., \& Avrett, E. H. 1989, Sol. Phys., 124, 303

Machado, M. E., Emslie, A. G., \& Brown, J. C. 1978, Sol. Phys., 58, 363

Machado, M. E., Avrett, E. H., Vernazza, J. E., \& Noyes, R. W. 1980, ApJ, 242, 336

Metcalf, T. R., Canfield, R. C., \& Saba, J. L. R. 1990, ApJ, 365, 391

Ricchiazzi, P. J., \& Canfield, R. C. 1983, ApJ, 272, 739

van Driel-Gesztelyi, L., Hudson, H. S., Anwar, B., \& Hiei, E. 1994, Sol. Phys., 152, 145

Vernazza, J. E., Avrett, E. H., \& Loeser, R. 1981, ApJS, 45, 635 